\title{
Well-to-Seismic Tie of a Field Onshore of the Nigerian Delta
}

\author{
1*INICHINBIA, S; ${ }^{2}$ SULE, PO \\ ${ }^{l}$ Department of Physics, University of Port Harcourt, Port Harcourt, Nigeria \\ ${ }^{2}$ Department of Physics, Ahmadu Bello University, Zaria, Nigeria \\ *Corresponding Author Email: sonny.inichinbia@yahoo.com; sonny.inichinbia@uniport-edu.ng
}

\begin{abstract}
This work presents a modern procedure for understanding seismic data wavelets through well-toseismic tie on an onshore field in the Nigerian Delta using the state-of-the-art techniques. The purpose of this work is the correlation of formation tops and seismic reflectors in the field. The objectives among others include the calibration of the seismic data in terms of polarity and phase, as well as to ensure that the seismic data is descriptive to well markers and discoveries, extending knowledge from the well location to rest of the field and reducing uncertainties. Logs from the two wells on the field and also logs from three wells on neighbouring fields were used to establish lateral continuity of the reservoirs H1000 and H4000. Their results show that the top, base and thickness of both reservoirs are quite variable laterally and this posed some challenges in the correlation from well to well. The field does not have checkshot data, so checkshot data from one of the wells on the neighbouring field was borrowed. Calibrated sonic and density logs of well01 and well-02 were used to assess the seismic ties at the well locations. Strong correlations at the wells are fundamental to the evaluation of the spatial extent of the horizons around the wells from the seismic data. Seismic-to-well ties are a very important part of the interpreter's business as they provide a means of correctly identifying horizons to pick, and estimating the wavelet for inverting seismic data to impedance and rock property indicators.
\end{abstract}

\section{DOI: $\underline{\text { https://dx.doi.org/10.4314/jasem.v25i1.7 }}$}

Copyright: Copyright (C) 2021 Inichinbia and Sule. This is an open access article distributed under the Creative Commons Attribution License (CCL), which permits unrestricted use, distribution, and reproduction in any medium, provided the original work is properly cited.

Dates: Received: 02 October 2020; Revised: 26 November 2020; Accepted: 12 December 2020

Keywords: Seismic, horizons, correlation, synthetics

The increased demands of quantitative interpretation methods demand an optimum tie between well and seismic data for any reservoir characterization workflow, whether amplitude variation with offset, impedance or neural network based. Quality well-toseismic tie does not come about by accident, but by careful editing, preconditioning/conditioning, calibration and timing among others, of the well data and the prestack seismic data. A seismic-to-well tie is the process through which seismic data wavelets are understood and compares seismic data at a well location with $\log$ data from the well. The idea is to obtain information on sediments, calibration of seismic processing parameters, correlation of formation tops and seismic reflectors, and derivation of a wavelet for seismic inversion among others (ElBehiry et al., 2020; Simm and White, 2002; White and Simm, 2003). From earlier times measurements had been made which include well-to-surface, surface-towell, and well-to-well measurements. Large offset surface-to-well measurements have been made to improve reflection seismic interpretation, to investigate anisotropy, and to localize faults or structures, in order to determine their elastic properties, among others. The well-to-well measurements were undertaken recently to obtain information on the elastic properties of reservoirs. However, this present article concerns modern wellto-seismic measurements, the objectives among others are the process by which wavelets are estimated through a well tie procedure that results in quantitative measures of synthetic-to-seismic tie and correlation of tops of horizons.

\section{MATERIALS AND METHOD}

The field was covered by a 3D seismic survey $(20 \mathrm{x}$ $17.5 \mathrm{~km}^{2}$ ) and the data was processed to anisotropic 3D full stack and three angle stacks (near angle stack, mid angle stack and far angle stack). The field also has well log data from two wells and has no checkshot data so the checkshot data was borrowed from a well in a neighbouring field. Other materials include Jason, techlog, petrel 2.0, savior and ArcGis.

Location of the Study Area: The field is situated in the Greater Ughelli depobelt of the Nigerian Delta. Figure 1 displays the map of the study area.

Well Data Availability: Figure 2 is a well panel displaying available data from the two wells (Well-01 and Well-02). The data quality was poor, and as such underwent thorough editing, conditioning, calibration, 
etc. Obvious data spikes were removed from the logs. Available logs include gamma ray, caliper, resistivity, density and sonic logs and borrowed checkshot. The drift curve was applied to the sonic log which was then integrated to give a time-depth relationship that fits the checkshot to within $1.0-2.0 \mathrm{~ms}$. The two panels on the right show a conventional display of sonic and density logs. Each panel contains both the logged raw data (blue) and the quantitative interpretation unit (QI) edited $\log$ (purple), with minimum and maximum ranges automatically adjusted to the data. The third panel from right displays the resistivity log for identification of fluid and lithology. The resistivity log is used as potential hydrocarbon indicator, spliced into a pseudo volume of clay $\left(\mathrm{V}_{\text {clay }}\right)$ curve derived from other curves. The resistivity log was normalized like most other logs, but the relationship with the volume of clay $\left(\mathrm{V}_{\text {clay }}\right)$ is most often not clear. For normalized resistivity above $0.5, \mathrm{~V}_{\text {clay }}$ was overwritten with resistivity to highlight potential pay zones and brine in this work, but other uses of resistivity logs include identification of tight streaks or volcanic intrusions. Since checkshot could be borrowed from neighboring wells or seismic/sonic velocities used without checkshot calibration, we decided to borrow checkshot data from a well on a neighbouring field.

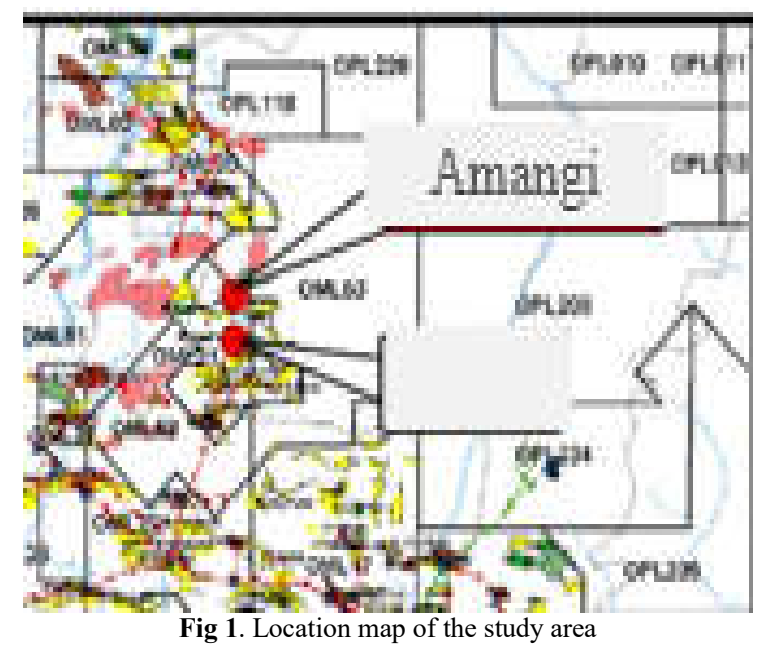

It is noteworthy that uncertainties in the well tie will increase under such circumstances, and significant corrections may be required and detailed velocity profiles usually require some smoothing to reduce excessive velocity contrasts at closely spaced timedepth pairs (Inichinbia, et al., 2014). Well log calibration brought the timing of the sonic log into agreement with the seismic times from the checkshot survey. Practical and theoretical analysis of the factors that influence the accuracy of well ties (White, 1997) shows that timing is paramount. Timing errors in the synthetic seismogram are much more detrimental than amplitude errors. They are especially damaging to estimating correctly the higher frequency components of the seismic wavelet. Well log calibration establishes the timing of the synthetic seismogram. Without it, the tie can only be fudged (White and Simm, 2003).

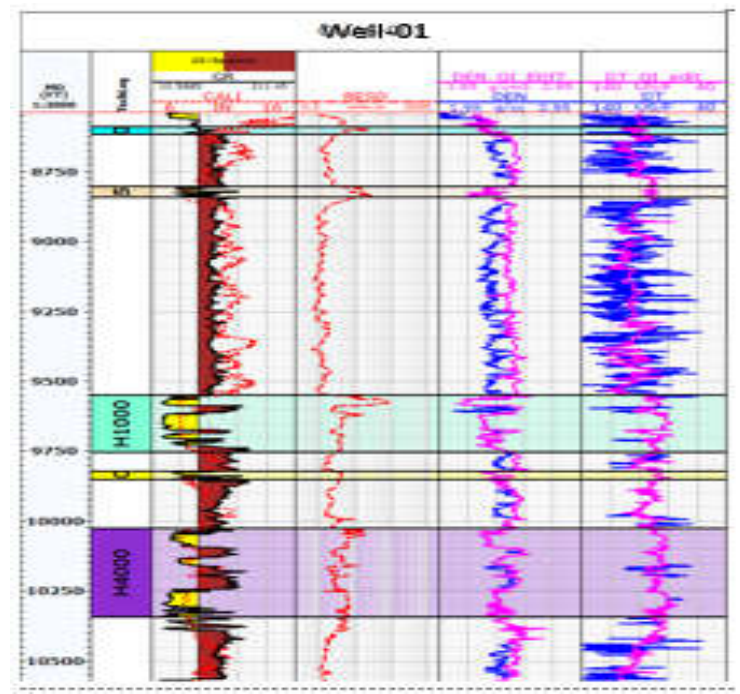

Fig 2. Well Log Editing

Seismic Data: Seismic data are recorded in time at a much lower resolution than the well data which are recorded in depth. Seismic data may be distorted by a complex overburden and therefore, migrated into an accurate image at the correct location. Errors in the 'migration' velocities could lead to lateral and vertical uncertainties in the seismic-to-well tie. Thus, a straightforward seismic-to-well tie comparison is hampered by the different nature of the data involved. The signature of the seismic imposes additional uncertainties. However, signal processing corrected for spherical divergence and absorption to obtain a stable response across an entire survey. The anisotropic 3D seismic data full volume, subdivided into the three angle stacks is displayed in Figure 3.

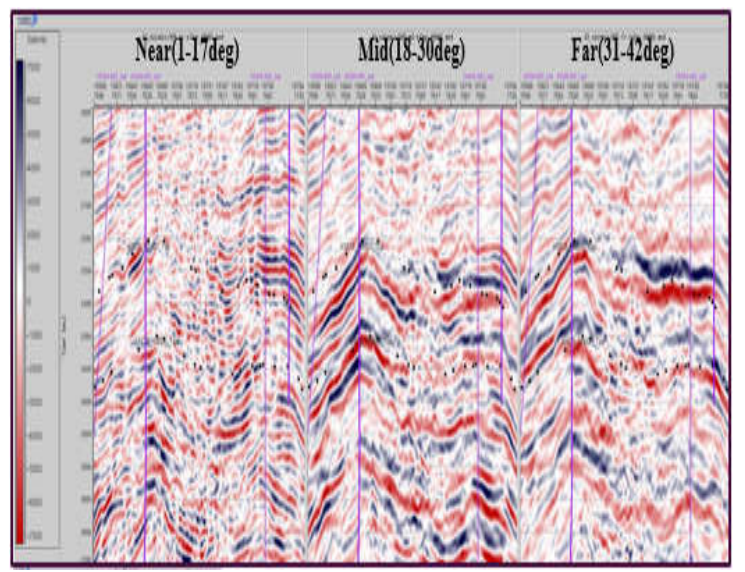

Fig 3. The three partial angle stacks used for the well-to-seismic tie 
With proper handling of the static and dynamic corrections and deconvolution, the stacking process improved the signal-to-noise ratio of the recorded data to such an extent that it becomes realistic to analyze amplitude and frequency characteristics of particular reflections. We used two seconds of the anisotropic 3D seismic reflection data and the synthetic to do the wellto-seismic tie. The well-to-seismic tie workflow adopted in this study is described in Figure 4.

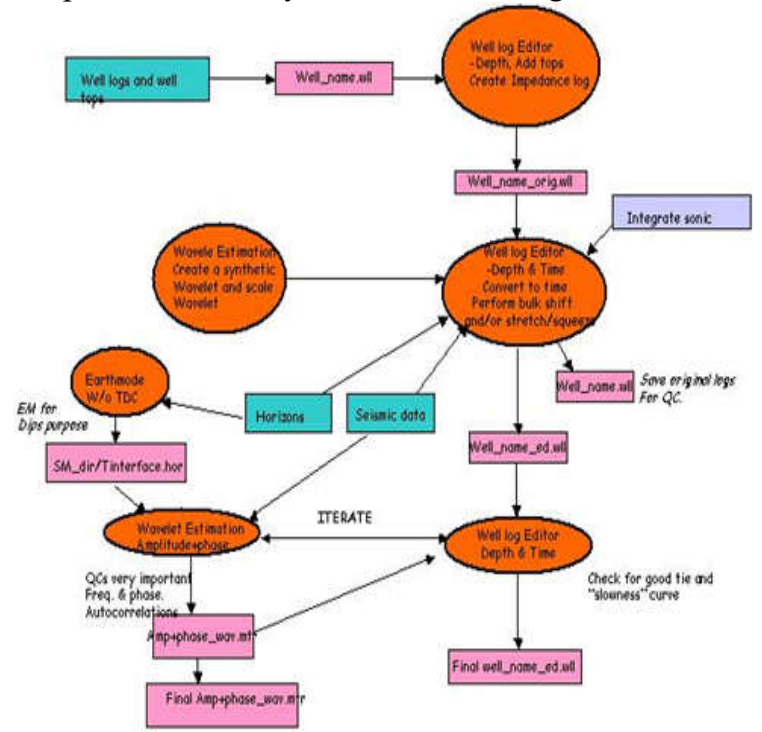

Fig 4. Simplified schematic workflow for Well tie to seismic and Wavelet Estimation
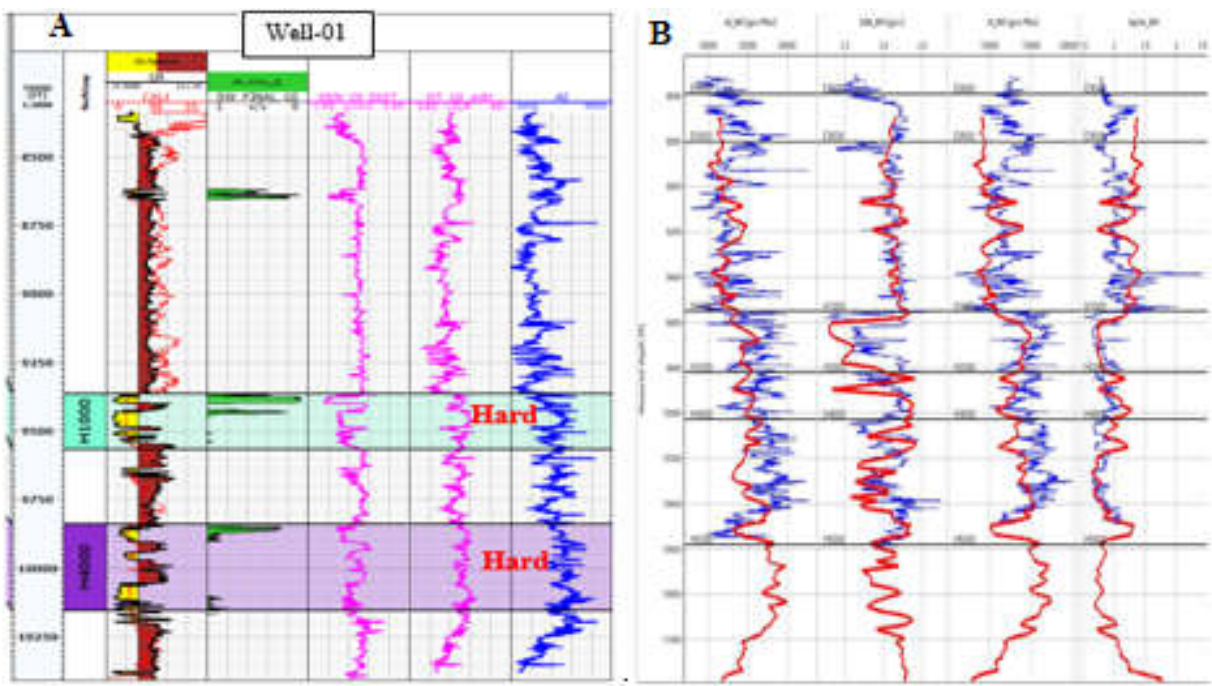

Fig 5. Log Signature of well-01, (a) acoustic impedance (AI) $\log$ (blue) and (b) shear impedance (SI).

Multi log displays are prone to clutter that masks coherent events. Normalization to a common shale level and variable density display softens the clutter and allows multiple logs to be arranged side by side in a default order of Gamma ray, Density, Neutron, Shear and Sonic, with some horizontal blending to diffuse contrasts, to produce a composite borehole image in a

\section{RESULTS AND DISCUSSION}

The panel on the extreme right of Figure 5(a) shows the acoustic impedance (AI) log (blue) of well-01 obtained by convolution of the QI edited sonic and the density logs in the second and third panels from the right respectively. Understanding acoustic log response is thus very important.

The second and third panels from the right display the sonic and density logs (purple) respectively, after adjustment of clipping ranges and reversal of the sonic $\log$. The coherency of the logs is more apparent, but it actually really tedious, if not impossible, to arrange all the data in a compatible manner. The Figure shows a gas sand and the top of the sands H1000 and H4000 are both hard reflectors.

The panel on the left of Figure 5(b) shows a shear impedance (SI) obtained by convolution a shear sonic with density $\log$ and a burial trend derived from a sliding median over $9000.0 \mathrm{ft}$ and a smooth spline. Normalization with the background yields a relative curve around a constant background of one. The anomalies on the $\operatorname{logs}$ correspond to gas sands (reservoirs) with sonic and shear velocities overlapping those of the bounding shale. The anomaly is retained in the transformation. 
resampled from reflection depth to reflection time. Convolution of the reflection time series with a seismic wavelet yields a synthetic seismogram that was used to compare with the actual seismic. All dips were flattened prior to the correlation to avoid inadvertent loss of high frequencies. Also, the impact of random noise was mitigated via cross correlation of neighbouring traces. The amplitude spectrum of the seismic was derived from auto correlations in an area around the borehole location. The spectrum was derived in a time gate of one second centred on the synthetic across all the traces in the selected traverse. The scaling of the seismic was determined from the median (or absolute) amplitude within the derive gate, and the dominant frequency was determined from the number of zero crossings per second. All seismic and synthetics are displayed in their respective panels of the ties.

Tieing Or Matching The Synthetic And The Seismic: Matching is usually performed on time migrated seismic data. A volume of data around a well was scanned for the best match location. For various reasons, the best match often does not occur at the well location. Since velocity typically increases with depth, time migration commonly moves the best match location up-dip from the well. A zero phase wavelet was estimated from the seismic amplitude spectrum. Convolution of this wavelet and the reflectivity time series from the well produced the synthetic, and the dominant frequency of the synthetic was compared with the dominant frequency of the seismic regardless of the phase and time shifts. The median (or absolute) amplitude of the synthetic was used to scale the wavelet. The seismic is compared against zero phase synthetics, resulting in a minimal phase difference as the polarity was the same. The best phase was obtained when the peak of the cross correlation coincided with the peak of the correlation envelope and the best seismic-to-well tie was obtained when the peaks are aligned without time shift, resulting in a correlation peak at zero and side lobes that are more balanced. The enhanced correlation of a perfect seismic-to-well tie, (i.e. the enhanced auto correlation), has a strong central lobe with rapidly declining tails and is skewed towards positive values. In order to obtain a more robust phase estimate, the skewness was derived across a series of time variant correlations, and also in a corridor of seismic traces around the borehole. The enhanced correlations were tapered beyond $50 \%$ of the maximum time lag, to avoid truncation errors. Timedepth errors within this $50 \%$ range are indeed reasonable. Larger errors, or dips within multi-trace corridors, might bias the phase estimate and so were avoided. Figures 6, 7 and 8 display the panels of the seismic-to-well ties of the three angle stacks the well- to-seismic ties. The panel on the extreme right hand side of these ties displays the QI edited and calibrated gamma ray (brown), sonic (purple) and acoustic impedance (blue) logs from left to right respectively. The coherency of the logs is apparent. It is noteworthy that a series of simple log transformations can normalize several logs to a common shale response, enabling coherent display of multiple logs in a single image. Such image enhances coherent contrasts to facilitate correlation with seismic events. So, these logs are arranged in a compatible manner, although they are not displayed in a single mage. The correlation panel contains important diagnostics to resolve time-depth and phase issues. The seismic-tosynthetic correlation of a proper seismic-to-well tie peaks at lag time zero and has balanced side lobes. This is clearly the case in these Figures. Synthetic traces from logs of well-01 are compared to traces from the 3D seismic data set. Logs in well-01 are the basis for comparison with the borrowed checkshot data. For all the three cases (ties) a time-to-depth conversion curve was obtained from direct arrival traveltimes measured on the borrowed zero offset checkshot. Figure 6 is the panel of the well-to-seismic ties of the near angle stack, and shows gas sands with zero phase synthetic, the seismic image is slightly shallower than the synthetic; a minor time-depth issue that was resolved with a static shift. In general, both the traces from the 3D seismic survey and those from the synthetic traces from the well show negative amplitude troughs at the top and positive peaks at the base of the H1000.

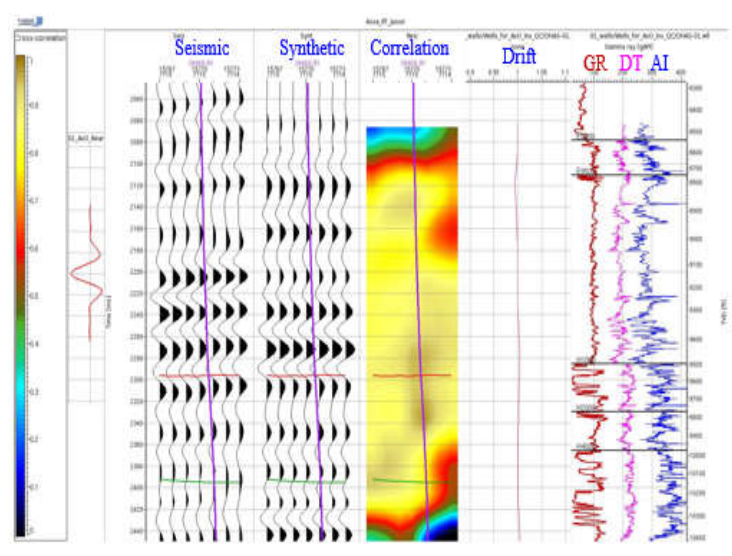

Fig 6. Well-to-seismic tie of the near angle stack

These two reflections are excellent markers to determine the spatial extent of the H1000 reservoir at the wells. On the other hand H4000 reservoir has positive amplitude peaks at the top and negative troughs at the base. Also, these two reflections are excellent markers to determine the spatial extent of the H4000 reservoir at the wells. The top of the sand 
H1000 (red horizon) is a hard reflector while the top of the sand H4000 (green horizon) is a soft reflector and the well tie appears simple. The traces of both the 3D seismic and the synthetic also predicted reflection within the reservoirs zones, corresponding to change or variations in P-wave velocity within these intervals. Both the 3D seismic traces and the synthetic traces reveal strong and continuous troughs at the top of horizon H1000 and weak peaks at the base, whereas the top of horizon H4000 revealed weak peaks and strong and continuous troughs at the base. In H1000, there is a weak trough and strong peak within the zone whereas there exists strong troughs and peaks within the H4000 (compare with Figures 2 and 5). These reflections correlated well with the synthetic traces.

Figure 7 displays the panel of the well-to-seismic tie of the mid angle stack, the seismic image was slightly shallower than the synthetic; which is a minor timedepth issue that was resolved with a static shift. The correlation panel contains important diagnostics to resolve time-depth and phase issues. Recall that the synthetic-to-seismic correlation of a proper well tie peaks at lag time zero and has balanced side lobes, and also, this is clearly the case in this tie. In fact, the correlations are predominantly positive, suggesting same polarity between the seismic and the synthetic. Although time variant stretch and squeeze can iron out small wrinkles, it alters the velocity profile of the timedepth table. So, excessive changes were avoided and where necessary, explained by anomalies in the vicinity of the well. Figure 8 is the panel showing the well-to-seismic tie of the far angle stack. The 3D seismic and synthetic traces do not correlate well in the time range corresponding to the horizon $\mathrm{H} 4000$ (green). In all the three ties, the synthetic traces at H1000 enjoy excellent correlation with the seismic traces and provide most of the details predicted on the synthetic traces. Such resolution is required to identify any variations within the reservoirs. The drift curve is applied to the sonic log which is then integrated to give a time-depth relationship that fits the checkshots to within $1.0-2.0 \mathrm{~ms}$. The picking accuracy of checkshot times is of the order of $1.0 \mathrm{~ms}$ and the calibration constrained the timing of the synthetic seismogram to a similar accuracy.

Thus, the well-to-seismic tie generated synthetics with compatible signature with the surface seismic to enable comparison with the seismic at the well location. The comparison resolves differences in velocity and phase, but may be hampered by noise as a result of the logs and synthetics impaired by borehole conditions. As such a better tie was achieved by tailoring the wavelet and synthetic to the seismic.

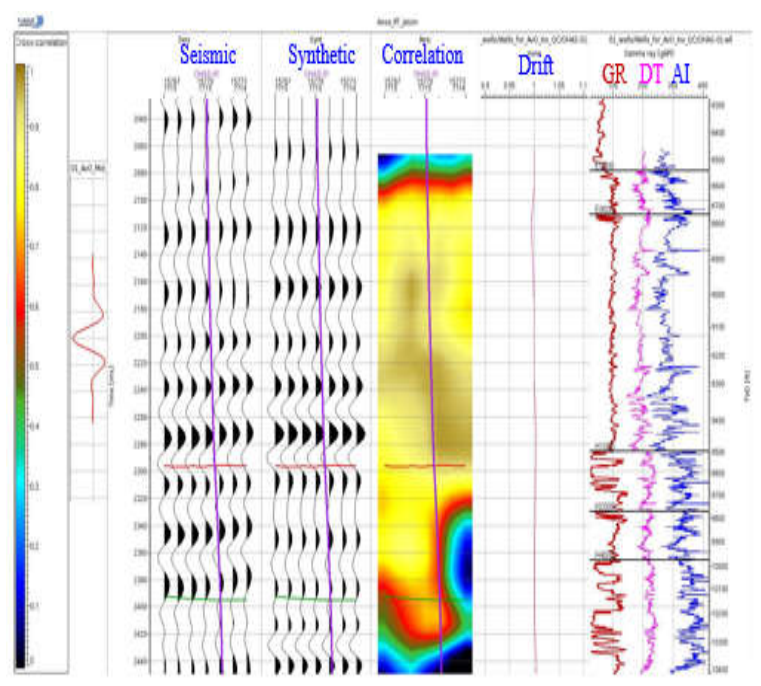

Fig 7. Well-to-seismic tie of the mid angle stack

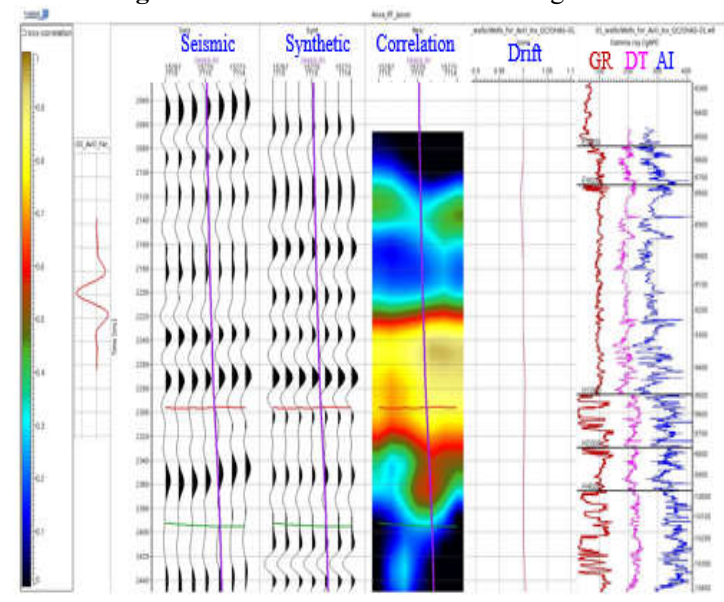

Fig 8. Well-to-seismic tie of the far angle stack

Wavelet Estimation: The 3D seismic data set was processed to zero phase to produce zero phase wavelet with frequencies of $10 \mathrm{~Hz}-60 \mathrm{~Hz}$, while the checkshot wavelet, though zero phase also, but has higher frequency content $(10 \mathrm{~Hz}-120 \mathrm{~Hz})$ and as such produced synthetic traces with higher vertical resolution (Bellefleur et al., 2006). The broadband reflectivity was filtered by the seismic wavelet in order to tie the well to the data. A problem in estimating the wavelet is that the transmission response of the earth has to be included if the filtered synthetic seismogram is to match the seismic data. The angle dependent wavelets were estimated from an acoustic impedance well $\log$ and a set of seismic traces, but White and Simm (2003), provides details of the approach to the estimation of the zero phase wavelet used in this tie. However, wavelet analysis is computing a filter, which best shapes the well log reflection coefficients to the input seismic at the well location. A good well tie over a long interval (few hundred milliseconds) is critical for a good wavelet. The best way to estimate wavelets is using well logs. 
Construction of Synthetics Seismogram: Constructing the synthetic involves creating a reflection series in time and convolving this with a wavelet. Synthetic modelling was done at well-01 using the 3D wavelet and well-01 calibrated density and P-wave sonic logs. Traces were extracted from the appropriate section of the 3D data set through well-01. The tine-to-depth conversion curve was derived from the first arrival traveltimes using a zero offset checkshot that was borrowed. Reflection coefficients were calculated from the calibrated density and P-wave velocity $\left(V_{p}\right)$ logs. Synthetic seismic traces for vertical incidence were calculated by convolving the reflection coefficients obtained from the calibrated sonic and density logs with the wavelet extracted from the anisotropic 3D seismic and the borrowed checkshot data sets using frequency matching. Internal multiples and other multiples were not used in the modelling of the synthetics but the primaries-only reflectivity (Bellefleur et al., 2006). The effect of imperfect migration velocities is less predictable and it is even possible that the well location is suspect. During the scanning procedure the choice of analysis parameters in estimating the wavelet can be investigated. The best match time location is found by scanning a number of traces around the well for goodness-of-fit using, say, a $500 \mathrm{~ms}$ segment of synthetic seismogram. Over such a segment it can be generally assumed that phase is not varying significantly. Reliability of the procedure deteriorates rapidly if shorter segments are used. When more than $500 \mathrm{~ms}$ of calibrated sonic is available the best time gate in which to establish the tie should also be investigated (White and Simm, 2003).

Conclusion: Synthetic modelling using the calibrated sonic and density logs reveals the top and base of horizons $\mathrm{H} 1000$ and $\mathrm{H} 4000$ can be identified on the 3D seismic sections as conspicuous isolated reflections. Other additional smaller scale layers could also be identified on the quality high resolution data. The industry processed 3D seismic data set preserved the relative true amplitudes.

Acknowledgements: The authors are thankful to Prahlad Basak, Dike Robinson, Francesca I. Osayande, Temitope Jegede, and the staff of the geosolutions and QI asset teams of the SPDC of Nigeria Limited. We also thank the SPDC of Nigeria Limited for providing the data.

\section{REFERENCES}

Bellefleur, G, Riedel, M and Brent, T (2006). Seismic characterization and continuity analysis of gas hydrate horizons near Mallik research wells, Mackenzie Delta, Canada. The Leading Edge. 25(5): $599-604$.

El-Behiry, MG, Al Araby, MS and Ragab, RZ (2020). Impact of phase rotation on reservoir characterization and implementation of seismic well tie technique for calibration offshore Nile Delta, Egypt. The Leading Edge. 39(5): 346 - 352.

Inichinbia, S, Sule, PO, Ahmed, AL and Hamza, H (2014). Well-to-seismic tie of Amangihydrocarbon field of the Niger Delta of Nigeria. J. Appl. Geol. Geophy. 2(2): Ver. I., 97 105.

Simm, R and White, RE (2002). Phase, polarity and the interpreter's wavelet, First Break. 20(5): 277281.

White, R and Simm, R (2003). Tutorial: Good practice in well ties. First Break. 21(5): 75 - 83.

White, RE (1997). The accuracy of well ties: practical procedures and examples, Expanded Abstract $R C 1.5,67$ th SEG Meeting, Dallas. 